\title{
The combination effects of trivalent gold ions and gold nanoparticles with different antibiotics against resistant Pseudomonas aeruginosa
}

\author{
Zeinab Esmail Nazari • Maryam Banoee • \\ Abbas Akhavan Sepahi • Fatemeh Rafii • \\ Ahmad Reza Shahverdi
}

Published online: 25 April 2012

(C) The Author(s) 2012. This article is published with open access at Springerlink.com

\begin{abstract}
Despite much success in drug design and development, Pseudomonas aeruginosa is still considered as one of the most problematic bacteria due to its ability to develop mutational resistance against a variety of antibiotics. In search for new strategies to enhance antibacterial activity of antibiotics, in this work, the combination effect of gold materials including trivalent gold ions $\left(\mathrm{Au}^{3+}\right)$ and gold nanoparticles (Au NPs) with 14 different antibiotics was investigated against the clinical isolates of $P$. aeruginosa, Staphylococcus aureus and Escherichia coli. Disk diffusion assay was carried out, and test strains were treated with the sub-inhibitory contents of gold nanomaterial. Results showed that Au NPs did not increase the antibacterial effect of antibiotics at tested concentration $(40 \mu \mathrm{g} / \mathrm{disc})$. However, the susceptibility of resistant $P$. aeruginosa increased in the presence of $\mathrm{Au}^{3+}$ and methicillin, erythromycin, vancomycin, penicillin G, clindamycin and nalidixic acid, up to $147 \%$. As an individual experiment, the same group of antibiotics was tested for their
\end{abstract}

\section{Z. E. Nazari}

Institute of Physics and Nanotechnology, Aalborg University,

Aalborg, Denmark

M. Banoee $\cdot$ A. A. Sepahi

Azad University of North Tehran Branch,

Tehran, Iran

F. Rafii

Division of Microbiology,

National Center for Toxicological Research,

Jefferson, AR, USA

A. R. Shahverdi $(\bowtie)$

Department of Pharmaceutical Biotechnology and Biotechnology

Research Center, Faculty of Pharmacy,

Tehran University of Medical Sciences,

Tehran, Iran

e-mail: shahverd@sina.tums.ac.ir activity against clinical isolates of $S$. aureus, E. coli and a different resistant strain of $P$. aeruginosa in the presence of sub-inhibitory contents of $\mathrm{Au}^{3+}$, where $\mathrm{Au}^{3+}$ increased the susceptibility of test strains to methicillin, erythromycin, vancomycin, penicillin $\mathrm{G}$, clindamycin and nalidixic acid. Our finding suggested that using the combination of sub-inhibitory concentrations of $\mathrm{Au}^{3+}$ and methicillin, erythromycin, nalidixic acid or vancomycin may be a promising new strategy for the treatment of highly resistant $P$. aeruginos $a$ infections.

Keywords $\mathrm{Au}^{3+} \cdot \mathrm{Au}$ NPs $\cdot$ Antibiotic resistance . Combination effect $\cdot$ Pseudomonas aeruginosa

\section{Introduction}

The emerging use of antibiotics has lead to microbial resistance which is still considered as a major problem in chemotherapy of many infectious diseases [1]. Many mechanisms are involved in the process of antibiotic resistances which mainly include enzymatic degradation and modification of the antibiotic agent [2], modification of the target site of the drug [3], and active reflux and reduced uptake of the drug [4]. Pseudomonas aeruginosa is an opportunistic human pathogen Gram negative bacterium which is responsible for infections such as blood stream nosocomial infections, especially in immunocompromised patients and the elderly population of industrial societies [5]. The organism has reputation for having minimal nutritional requirements, the ability to tolerate a wide variety of physical conditions and the ability to resist against new antibiotics such that it has been addressed as "the worst nightmare" of microbiologists and clinical pharmacists for its highly resistant nature [6]. The antibiotic resistance of 
this microorganism is due to a number of mechanisms which mainly include co-operation of multidrug efflux pumps such as MexAB-OprM, a pump system that removes $\beta$-lactams, chloramphenicol, fluoroquinolones, macrolides, novobiocin, sulfonamides, tetracycline and trimethoprim, as well as various dyes and detergents [7]; low impermeability of the membrane to drugs and the fact that it readily develops mutational resistance to most antibacterial agents [8]. The problem of multidrug resistance of $P$. aeruginosa has urged many scientists and pharmaceutical companies to search for new potential therapies for this Gram negative bacterium [9-11]. Therefore, studies on new strategies to combat $P$. aeruginosa would be of great value.

An alternative strategy to overcome the problem of resistance is the use of commonly used antibiotics in combination with different natural or chemical agents $[12,13]$. To date, many organic and inorganic compounds have been reported to enhance the antibacterial activity of different antibiotics against many bacteria and fungi resistant test strains [14]. Metallic ions and metallic nanoparticles including zinc and silver have particularly shown promise when used in combination with a number of antibiotics such as ciprofloxacin [15], penicillin G, amoxicillin, erythromycin, clindamycin, vancomycin [16], ampicillin, kanamycin, erythromycin and chloramphenicol [17] in different Gram positive or Gram negative test strains. In a recent approach, Rai et al. reported the antibiotic mediated synthesis of $\mathrm{Au}$ NPs with potent antimicrobial activity for application in antimicrobial coatings [18].

During our previous attempts to explore new agents that modulate antibiotic resistant, we screened different chemical substances and natural products and reported a number of organic and inorganic compounds which reduced the resistance of various Gram positive and negative bacteria including Staphylococcus aureus, Clostridium difficile and Aspergillus sp. [14, 19]. In particular, our team reported that some monoterpenes (one of the major components of essential oils) enhanced antibacterial activity of nitrofurantoin against resistant strains of Enterobacteria [19]. We also showed that the antibacterial activity of fluconazol against different species of Aspergillus increased in the presence of different concentrations of Sarcococca saligna ethanol extract [14]. In our very recent work, we demonstrated the enhanced activity of ciprofloxacin in the presence of $\mathrm{ZnO}$ nanoparticles [15]. We also reported that silver nanoparticles enhance antibacterial activity of different antibiotics against $S$. aureus and Escherichia coli [16]. However, our search for finding new compounds that enhance the activity of antibiotics against $P$. aeruginosa was not successful during past years.

To date, gold-based drugs have shown great promise in treatment of various diseases such as auranofin for the treatment of arthritis and triphenylphosphinegold (I) complexes for the treatment of cancer tumors, psoriasis and HIV infections [20]. Moreover, gold complexes have shown considerable cytotoxic and antimicrobial activity [21-23]. In this study, we report the combination effect of $\mathrm{Au}^{3+}$ on antibacterial activity of different antibiotics against clinically resistant strains of $P$. aeruginosa, $S$. aureus and E. coli.

\section{Material and methods}

Gold materials

Chloroauric acid was purchased from Merck, Darmstadt, Germany. Au NPs used during this investigation were prepared by previously described tannin-free ethanol extract of black tea (Camellia sinensis) method [24]. Briefly, an aqueous chloroauric acid solution $\left(10^{-3} \mathrm{M}\right)$ was added separately to the reaction vessel containing the tannin-free ethanol extract of black tea $(10 \% \mathrm{v} / \mathrm{v})$, and the resulting mixture was allowed to stand for $15 \mathrm{~min}$ at room temperature. The reduction of the $\mathrm{Au}^{3+}$ ions by tannin-free ethanol extract of black tea in the solutions was monitored by sampling the aqueous component $(2 \mathrm{ml})$ and measuring the UV-visible spectrum of the solutions. This sample was diluted three times with distilled water, and the UV-visible spectrum of this sample was measured on a Labomed Model UVD-2950 UV-Vis Double Beam PC Scanning spectrophotometer, operated at a resolution of $2 \mathrm{~nm}$. Furthermore, Au NPs were characterized by transmission electron microscopy (model EM 208 Philips). The gold colloid solution was centrufuged $(12000 \times g)$ for $60 \mathrm{~min}$. Subsequently the setteled Au NPs were washed three times with deionised water. A stock colloid solution $(100 \mathrm{mg} / \mathrm{ml})$ was prepared and reserved in $4^{\circ} \mathrm{C}$ for further experiments.

\section{Antimicrobial assay}

The antibacterial activity of $\mathrm{Au}^{3+}$ and $\mathrm{Au}$ NPs was evaluated at different contents $(31.25,62.5,125,250,500,1000,2000$ and $4000 \mu \mathrm{g} / \mathrm{disc}$ ) on Müeller-Hinton agar (MHA) (Difco, Germany) using conventional disk diffusion method against $P$. aeruginosa, $S$. aureus and E. coli. Minimum inhibitory content was defined as the lowest content of $\mathrm{Au}^{3+}$ or $\mathrm{Au}$ $\mathrm{NPs}$ creating clear zone of inhibition after $24 \mathrm{~h}$ at $35^{\circ} \mathrm{C}$. This disk diffusion susceptibility test was also carried out on MHA plates in order to examine the antibacterial activity of candidate antibiotics against resistant test strains. Standard antibiotics disks, listed in Table 1, were purchased from Mast Co., UK. In order to explore a possible combination effect of gold materials and antibiotics, each standard paper disk was impregnated with the sub-inhibitory content of $40 \mu \mathrm{g} /$ disk of $\mathrm{Au}^{3+}$ and $\mathrm{Au}$ NPs. A resistant strain of $P$. aeruginosa was obtained from Imam University Hospital (Tehran, Iran) and identified by conventional microbiological 
Table 1 The antibacterial activity of $\mathrm{Au}^{3+}$ ions and gold nanoparticles against Pseudomonas aeruginosa, Staphylococcus aureus and Escherichia coli

\begin{tabular}{llll}
\hline Compounds $(\mu \mathrm{g} / \mathrm{disk})$ & \multicolumn{3}{l}{ Zone of inhibition diameter $(\mathrm{mm})$} \\
\cline { 2 - 4 } & E. coli & P. aeruginosa $(\mathrm{I})$ & S. aureus \\
\hline $\mathrm{Au}^{3+}$ & & & \\
4000 & 32 & 30 & 34 \\
2000 & 28 & 24 & 30 \\
1000 & 21 & 20 & 22 \\
500 & 14 & 17 & 16 \\
250 & 10 & 14 & 13 \\
125 & 9 (MIC) & 12 & 11 \\
62.5 & - & 9 (MIC) & 9 (MIC) \\
31.25 & - & - & - \\
$\mathrm{AU}$ NPs & & & 13 \\
4000 & 14 & 14 & 10 (MIC) \\
2000 & 10 & 11 & - \\
1000 & 9 (MIC) & 9 (MIC) & - \\
500 & - & - & - \\
250 & - & - & - \\
125 & - & - & - \\
62.5 & - & - & - \\
31.25 & - & - & - \\
\hline
\end{tabular}

Minimum inhibitory content (MIC) was defined as the lowest content of $\mathrm{Au}^{3+}$ or Au NPs creating clear zone of inhibition after $24 \mathrm{~h}$ at $35^{\circ} \mathrm{C}$

and biochemical methods. A single colony of test strains was grown overnight on Mueller-Hinton broth (MHB) medium on a rotator shaker $(200 \mathrm{rpm})$ at $35^{\circ} \mathrm{C}$. The inocula were prepared by diluting the cultures with $0.9 \% \mathrm{NaCl}$ to a $0.5 \mathrm{McF}$ arland standard and were applied to the plates along with the standard and test disks containing $40 \mu \mathrm{g} /$ disk of $\mathrm{Au}^{3+}$ and $\mathrm{Au}$ NPs. After incubation at $35^{\circ} \mathrm{C}$ for $24 \mathrm{~h}$, the zones of inhibition were measured. The mean surface area of each inhibition zone (square millimeter) was calculated from the mean diameter of each tested antibiotic. The percent of increase in the inhibition zone areas for different antibiotics against $P$. aeruginosa was calculated as $\left(b^{2}-a^{2}\right) / a^{2} \times 100$ where $a$ is the inhibition zone in the presence of antibiotic only, and before addition of $\mathrm{Au}^{3+}$ and Au NPs, while $b$ represents the inhibition zone in the presence of antibiotic plus $\mathrm{Au}^{3+}$ or $\mathrm{Au}$ NPs. The same procedure was used for combination of $\mathrm{Au}^{3+}$ and $\mathrm{Au}$ NPs with antibiotics against additional test strains. All experiments were performed in triplicate.

Additionally, a different clinical isolate of $P$. aeruginosa and two test strains of $S$. aureus and E. coli were obtained from Ghods Polyclinic Laboratory (Tehran, Iran), and the same procedure was repeated. To compare the antibacterial activity results of test and control samples and in order to avoid possible errors, a parallel test was run for pure antibiotics with conditions similar to those for antibiotic-gold material combination. The enhancing effect of $\mathrm{Au}^{3+}$ with different antibiotics was further determined against mentioned test strains using the method described above (Table 1).

\section{Results and discussion}

In this study, the Au NPs were prepared using a tannin-free ethanol extract of black tea. The inset to Fig. 1 shows the tubes containing this tannin-free extract before (tubes A) and after the reaction with $\mathrm{Au}^{3+}$ for 15 min (tubes B). The gold-containing solutions (tubes A and B) that were a transparent yellow at first turned into purple on completion of the reaction by the tannin-free ethanol extract of black tea (tube B). These reaction mixtures were further characterized by UV-visible spectroscopy. As illustrated in Fig. 1, a strong surface plasmon resonance maximum was observed at ca. $527 \mathrm{~nm}$. This peak is assigned to a surface plasmon phenomenon that is well documented for various metal nanoparticles with sizes ranging from 2 to $100 \mathrm{~nm}$ [25-27]. Figure 2 shows representative TEM images recorded from the drop-coated film of the as-prepared Au NPs, synthesized by treating the chloroauric acid solution with a tannin-free ethanol extract of black tea (left picture) after $15 \mathrm{~min}$. The particle size histogram of these spherical gold particles, produced with this tannin-free ethanol extract (right illustration) in Fig. 2), shows that the particles range in size from 1.25 to $17.5 \mathrm{~nm}$. It should be mentioned that almost $60 \%$ of prepared Au NPs were in the range 2-6 nm.

The antibacterial effect of $\mathrm{Au}^{3+}$ and Au NPs against $P$. aeruginosa, $S$. aureus and $E$. coli has been determened by

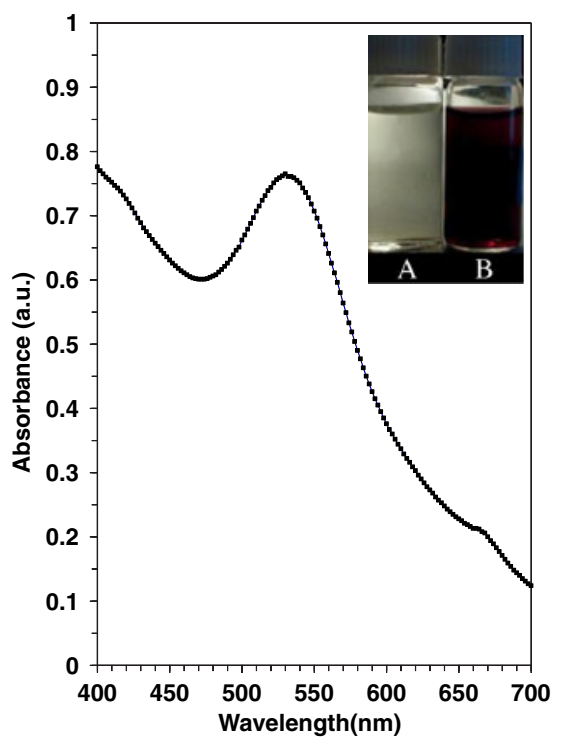

Fig. 1 UV-visible spectrum of gold colloid. Spectrum recorded after adding the tannin-free ethanol extract of black tea $(10 \mathrm{ml})$ to $90 \mathrm{ml}$ of a chloroauric acid solution $(1 \mathrm{mM})$. The curve is recorded after a period of $15 \mathrm{~min}$. The inset shows the solution of chloroauric acid $(1 \mathrm{mM})$ before (a) and after exposure to the tannin-free ethanol extract of black tea (b) 
Fig. 2 Transmission electron micrograph recorded from a small region of a drop-coated film of chloroauric acid solution treated with the tannin-free ethanol extract of black tea (leftside picture) for $15 \mathrm{~min}$ (scale bars correspond to $50 \mathrm{~nm}$ ). The related particle size distribution histogram (right-side picture) obtained after measuring the size of 350 individual particles
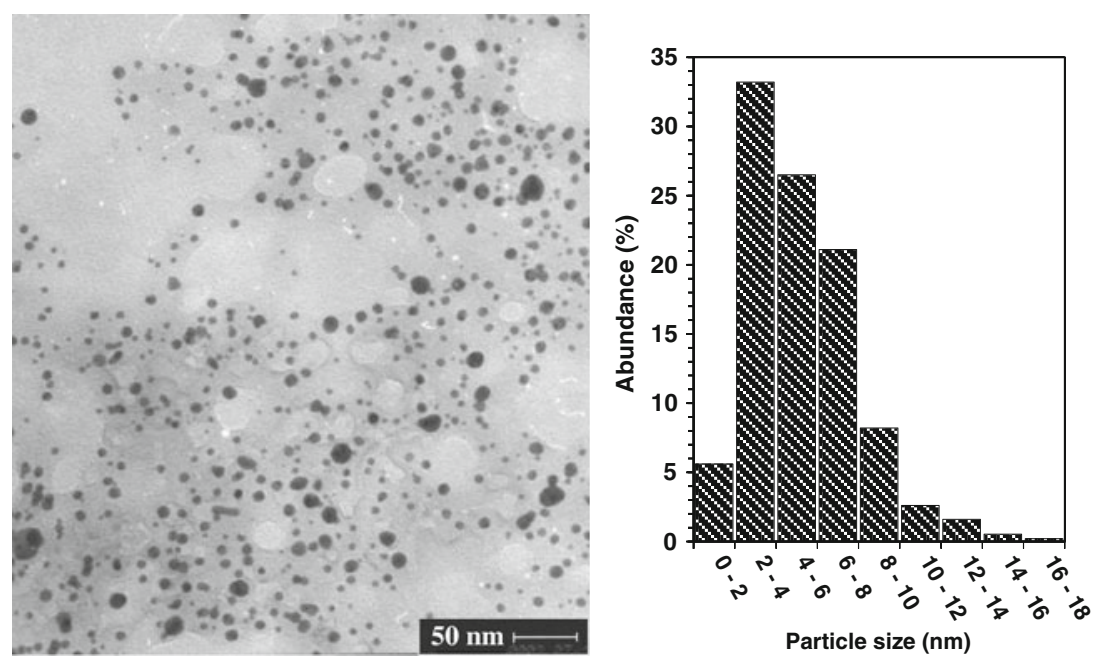

disk diffusion method and reported in Table 1. Higher concentrations of both $\mathrm{Au}^{3+}$ and $\mathrm{Au}$ NPs $(1000-4000 \mu \mathrm{g} / \mathrm{disc})$ showed antibacterial activity aganist above test strains. As antibacterial agent, the $\mathrm{Au}^{3+}$ ions were considarably more potent than inert $\mathrm{Au}$ NPs against all test strians. Lowest MICs were obtained for $\mathrm{Au}^{3+}$ against $P$. aeruginosa, $S$. aureus and $E$. coli (Table 1). In the next step, the antibacterial activity of sub-inhibitory contents of $\mathrm{Au}^{3+}$ and $\mathrm{Au}$ NPs was investigated in combination with a number of commonly used antibiotics against resistant strains of $P$. aeruginosa, $S$. aureus and E. coli. The diameters of inhibition zones (millimeter) in antibiotic disks both in the presence and absence of sub-inhibitory contents of $\mathrm{Au}^{3+}$ and $\mathrm{Au}$ NPs were calculated. It should be noted that no antibacterial activity was observed for both $\mathrm{Au}^{3+}$ and $\mathrm{Au}$ NPs at concentartions lower than $62.5 \mu \mathrm{g} /$ disk. In this investigation the sub-inhibitory content of $40 \mu \mathrm{g} / \mathrm{disk}$ was chosen which is much lower than the MIC value required to produce antibacterial effect. Therefore, any increase in the antibacterial effect of antibiotics could be attributed not to the cytotoxic effect of $\mathrm{Au}^{3+}$, but to the combination effect of $\mathrm{Au}^{3+}$ with antibiotics.

Table 2 shows the inhibition zones (square millimeter) of candidate antibiotics against two different strains of $P$. aeruginosa both in presence and absence of sub-inhibitory content of $40 \mu \mathrm{g} / \mathrm{disk} \mathrm{Au}^{3+}$. As shown in the Table 2, different antibiotics showed different activities in the presence of $\mathrm{Au}^{3+}$. In both resistant $P$. aeruginosa isolates, the antibacterial activity of penicillin G, methicillin, erythromycin, vancomycin, clindamycin and nalidixic acid increased, while no enhancing effect was observed for the remaining antibiotics. In detail, the surface area of inhibition zones (percent) in resistant $P$. aeruginosa strain 1 plates containing either of methicillin, erythromycin, vancomycin, penicillin $\mathrm{G}$, clindamycin and nalidixic acid increased by 147,147 , 147, 104, 125 and $147 \%$, respectively.

Furthermore, the effect of $\mathrm{Au}^{3+}$ was evaluated using the same set of antibiotics against a different clinical isolate of
$P$. aeruginosa and the clinical isolates of $S$. aureus and $E$. coli. In $P$. aeruginosa strain 2 group, the sensitivity of the

Table 2 Increase in inhibition zone area (\%) of candidate antibiotics against two resistant test strains of Pseudomonas aeruginosa, the clinical isolates of Staphylococcus aureus and Escherichia coli in the presence of $\mathrm{Au}^{+3}$ at sub-inhibitory content of $40 \mu \mathrm{g} / \mathrm{disk}$

\begin{tabular}{|c|c|c|c|c|}
\hline \multirow{2}{*}{$\begin{array}{l}\text { Antibiotics } \\
(\mu \mathrm{g} / \text { disk })\end{array}$} & \multicolumn{4}{|c|}{ Increase in inhibition zone area $(\%)$} \\
\hline & $\begin{array}{l}\text { P. aeruginosa } \\
\text { (1) }\end{array}$ & $\begin{array}{l}\text { P. aeruginosa } \\
\text { (2) }\end{array}$ & $\begin{array}{l}S . \\
\text { aureus }\end{array}$ & $\begin{array}{l}\text { E. } \\
\text { coli }\end{array}$ \\
\hline Penicillin G 10 & 104 & 65 & 19 & 0 \\
\hline Amoxicillin 10 & 0 & 0 & 0 & 0 \\
\hline Methicillin 5 & 147 & 146 & 0 & 0 \\
\hline Cephalexin 30 & 0 & 0 & 0 & 30 \\
\hline Cefixime 5 & 0 & 0 & 0 & 0 \\
\hline $\begin{array}{l}\text { Erythromycin } \\
5\end{array}$ & 147 & 104 & 0 & 0 \\
\hline Gentamicin 10 & 0 & 0 & 7 & 0 \\
\hline Amikacin 30 & 0 & 0 & 7 & 9 \\
\hline Tetracycline 30 & 0 & 0 & 10 & 7 \\
\hline $\begin{array}{l}\text { Ciprofloxacin } \\
5\end{array}$ & 0 & 0 & 7 & 0 \\
\hline Clindamycin 2 & 125 & 65 & 13 & 65 \\
\hline $\begin{array}{l}\text { Nitrofurantoin } \\
300\end{array}$ & 0 & 0 & 8 & 21 \\
\hline $\begin{array}{l}\text { Nalidixic acid } \\
30\end{array}$ & 147 & 146 & 39 & 0 \\
\hline $\begin{array}{l}\text { Vancomycin } \\
30\end{array}$ & 147 & 104 & 0 & 147 \\
\hline
\end{tabular}

Mean surface area of the inhibition zone $\left(\mathrm{mm}^{2}\right)$ was calculated from the mean diameter of each tested antibiotic. The percent of increase in the inhibition zone areas in presence of sub-inhibitory contents of $\mathrm{Au}^{+3}$ for different antibiotics against Pseudomonas aeruginosa was calculated as $\left(b^{2}-a^{2}\right) / a^{2} \times 100$ where $a$ is the inhibition zone in the presence of antibiotic only, and before inoculation of $\mathrm{Au}^{3+}$, and $b$ represents the inhibition zone in the presence of antibiotic plus $\mathrm{Au}^{3+}$. No significant inhibition was observed when the combination of antibiotic-Au NPs was used under similar condition 
new isolate of $P$. aeruginosa to methicillin, erythromycin, vancomycin, penicillin $\mathrm{G}$, clindamycin and nalidixic acid increased by 146, 104, 104, 65, 65 and $146 \%$, respectively. However, the inhibition zone was not the same in different clinical isolates of P. aeruginosa (104\% and $65 \%)$. In $S$. aureus group, $\mathrm{Au}^{3+}$ slightly enhanced the antibacterial activity of penicillin G, gentamicin, amikacin, tetracycline, ciprofloxacin, clindamycin, nitrofurantoin and nalidixic acid. The most enhancing effects in this group were observed for nalidixic acid (39\% increase) and clindamycin (13\% increase). In E. coli group, $\mathrm{Au}^{3+}$ had enhancing effect on antibacterial activity of cephalexin, amikacin, tetracycline, clindamycin, nitrofurantoin and vancomycin, and the most enhancing effect was observed for cephalexin (30\% increase), clindamycin (65\% increase), nitrofurantoin ( $21 \%$ increase) and vancomycin (147\% increase).

The test was also repeated with the same set of antibiotics, using the same sub-inhibitory content of $40 \mu \mathrm{g} /$ disk Au NPs against resistant $P$. aeruginosa which showed that Au NPs did not have a significant effect on antibacterial activity of antibiotics at a content level of $40 \mu \mathrm{g}$ (results not shown).

In 2007, Grace and Pandium reported that Au NPs did not have antibacterial effects against a number of microorganisms including P. aeruginosa, S. aureus and E. coli, while coating of $\mathrm{Au}$ NPs with antibiotics increased their antibacterial activity [28]. Furthermore, recent studies by Burygin et al. revealed that Au NPs have no enhancing effect on the antibacterial activity of gentamycin [26]. In this study, no significant difference was observed between antibacterial activity of antibiotics alone and their mixture with Au NPs. However, as suggested by Burygin et al., it seems that Au NPs enhance antibacterial activity of antibiotics only when antibiotic is chemically attached on the surface of Au NPs and formed stable conjugates with particles rather than when used in combination with antibiotics as a mixture [29]. Therefore, comparing $\mathrm{Au}$ NPs and $\mathrm{Au}^{3+}$, it is plausible that $\mathrm{Au}^{3+}$ form more potent mixtures with antibiotics concerning the fact that they are more actively involved in reactions due to their ionic nature. This probably best explains our finding that when used together with antibiotics, Au NPs had no significant effect on antibacterial activity of antibiotics, while $\mathrm{Au}^{3+}$ (which form more potent complexes) enhanced the inhibition zone of bacterial growth.

Comparing four groups, it seems that the enhancing effect of $\mathrm{Au}^{3+}$ was more considerable in Gram negative $P$. aeruginosa and E. coli rather than Gram positive S. aureus. It is notable that studies of Marques et al. on MIC (minimum inhibitory concentration) value of $\mathrm{Au}^{3+}$ metal compounds in complex with sulphamethoxazole in $P$. aeruginosa, E. coli and $S$. aureus suggested no difference between the response of Gram positive and Gram negative bacteria treated with $\mathrm{Au}$ compounds in combination with sulphamethoxazole [30], while other studies suggest that the enhancing effect of $\mathrm{Au}^{3+}$ is better in Gram negative bacteria [31]. However, more investigations should be carried out at the molecular level to clarify whether $\mathrm{Au}^{3+}$ is more effective on Gram negative rather than Gram positive bacteria.

Fourteen candidate antibiotics were carefully chosen since they represent major classes of antibiotics (penicillins, cephallosporins, macrolides, aminoglicosides, tetracyclines, fluoroquinolones, lincomycin derivatives, nitrofurans and glycopeptides). However, different antibiotics showed different activities in the presence of $\mathrm{Au}^{3+}$. In P. aeruginosa, a considerable enhancing effect was observed for methicillin, erythromycin, vancomycin, penicillin $G$, clindamycin and nalidixic acid, while in $S$. aureus $\mathrm{Au}^{3+}$ slightly enhanced the antibacterial activity of penicillin $\mathrm{G}$, gentamicin, amikacin, tetracycline, ciprofloxacin, clindamycin, nitrofurantoin and nalidixic acid. In E. coli group, $\mathrm{Au}^{3+}$ had an enhancing effect on antibacterial activity of cephalexin, amikacin, tetracycline, clindamycin, nitrofurantoin and vancomycin.

It is already known that $\mathrm{Au}^{3+}$ may form a coordination complex with available donor groups such as nitrogen, sulfur and phosphor. Therefore, in the presence of antibiotics such as penicillin, nalidixic acid and clindamycin, $\mathrm{Au}^{3+}$ may form coordination complexes with ring nitrogen on these compounds. This interaction may have induced changes in morphology of these compounds, thereby increasing their efficiency. The same mechanism has been proposed for the interaction of gold (III) with zeatin [32]. In beta-lactam and cephalosporins such as penicillin and methicillin, this coordination may have happened between $\mathrm{Au}^{3+}$ and free electrons on sulphur and nitrogen donor groups. However, this was not the case for all beta-lactam and cephalosporins tested (amoxicillin and cefixime).

It is also deducible that the enhancing effect of $\mathrm{Au}^{3+}$ was more significant in Gram negative P. aeruginosa and E. coli rather than the Gram positive $S$. aureus. This is potentially interesting since studies by Chudasama et al. on core-shell silver nanostructures [33] and Nomiya et al. on gold (I) complexes [21] also revealed that gold complexes are considerably more effective on Gram negative rather than Gram positive bacteria. However, further investigations are suggested to be carried out on other Gram positive and Gram negative strains to see whether the structure of cell wall in bacteria affects the enhancing effect of gold materials.

So far, a number of elements including copper, lead and zinc have been studied for their interaction with $P$. aeruginosa $[28,34]$. As mentioned earlier, the antibiotic resistance of $P$. aeruginosa is primarily due to the co-operation of multidrug efflux pumps and impermeability of bacterial membrane [6]. Therefore, it is possible that $\mathrm{Au}^{3+}$ may sensitize $P$. aeruginos $a$ cells by either interfering in the function of these efflux pumps or increasing the permeability of the bacterial membrane. Moreover, P. aeruginosa is well known for its resistance to heavy metals through reduction of metal 
ions. For instance, it actively resists against $\mathrm{Au}^{3+}$ through reducing it to its metallic form $[35,36]$. However, when treated with the combination of antibiotic- $\mathrm{Au}^{3+}, P$. aeruginosa was more sensitive to the antibiotics. This could be due to the possible new interactions that may form between antibiotics and $\mathrm{Au}^{3+}$ which literally hinder $P$. aeruginosa from reducing it to metallic $\mathrm{Au}$ and the fact that the organism may not be able to reduce heavy metal ions in the presence of antibiotics. However, more experiments are required to be done in order to determine the underlying mechanism of $\mathrm{Au}^{3+}$ in the presence of antibiotics. The mechanism underlying the enhancing effect of $\mathrm{Au}^{3+}$ is probably multi-factorial, and a molecular approach is necessary to identify the determinants of this effect.

\section{Conclusion}

The result of this work demonstrates that using $\mathrm{Au}^{3+}$, it is possible to enhance the efficacy of a number of commonly used antibiotics against $P$. aeruginosa up to $146 \%$. This finding is of particular value since resistant strains of $P$. aeruginosa are considered as a major problem in chemotherapy of many infectious diseases. This enhancing effect also occurred in other resistant microorganisms including $S$. aureus and E. coli. This suggests that the combination therapy of gold materials such as $\mathrm{Au}^{3+}$ could be considered as a new approach and the common antibiotics may have an even broader range of medical applications in the future.

Acknowledgments This work was supported by the Pharmaceutical Sciences Research Center, Faculty of Pharmacy, Tehran University of Medical Sciences, Tehran, Iran. The views presented in this article do not necessarily reflect those of the U.S. Food and Drug Administration.

Open Access This article is distributed under the terms of the Creative Commons Attribution License which permits any use, distribution and reproduction in any medium, provided the original author(s) and source are credited.

\section{References}

1. Levy SB, Marshall B (2004) Antibacterial resistance worldwide: causes, challenges and responses. Nat Med 10:122-129

2. Wright GD (2005) Bacterial resistance to antibiotics: enzymatic degradation and modification. Adv Drug Deliver 57:1451-1470

3. Lambert PA (2005) Bacterial resistance to antibiotics: modified target sites. Adv Drug Deliver 57:1471-1485

4. Kumar A, Schweizer HP (2005) Bacterial resistance to antibiotics: active efflux and reduced uptake. Adv Drug Deliver 57:1486-1513

5. Wisplinghoff $\mathrm{H}$ et al (2003) Current trends in the epidemiology of nosocomial bloodstream infections in patients with hematological malignancies and solid neoplasms in hospitals in the United States. Clin Infect Dis 36:1103-1110

6. Livermore DM (2002) Multiple mechanisms of antimicrobial resistance in Pseudomonas aeruginosa: our worst nightmare? Antimicrobial Resistance 34:634-640
7. Poole K (2001) Multidrug efflux pumps and antimicrobial resistance in Pseudomonas aeruginosa and related organisms. J Mol Microbiol Biotechnol 3:255-264

8. Li XZ, Zhang L, Poole K (2000) Interplay between the MexAMexB-OprM multidrug efflux system and the outer membrane barrier in the multiple antibiotic resistance of Pseudomonas aeruginosa. J Antimicrob Chemother 45:433-436

9. Cornelis P (2008) Pseudomonas: genomics and molecular biology. Caister Academic, Brussels

10. Poole K (2004) Efflux-mediated multiresistance in Gram-negative bacteria. Clin Microbiol Infec 10:12-26

11. Obritsch MD et al (2005) Nosocomial infections due to multidrugresistant Pseudomonas aeruginosa: epidemiology and treatment options. Pharmacotherapy 25:1353-1364

12. Tan YT, Tillett DJ, Mackay LA (2000) Molecular strategies for overcoming antibiotic resistance in bacteria. Mol Med Today $6: 309-314$

13. Wright GD (2000) Resisting resistance: new chemical strategies for battling superbugs. Chem Biol 59:7-16

14. Mollazadeh Moghaddam K et al (2010) The Antifungal activity of Sarcococca saligna ethanol extract and its combination effect with fluconazole against different resistant Aspergillus species. Appl Biochem Biotech 162(1):127-133

15. Banoee $\mathrm{M}$ et al (2010) $\mathrm{ZnO}$ nanoparticles enhanced antibacterial activity of ciprofloxacin against Staphylococcus aureus and Escherichia coli. J Biomed Matter Res B 93:557-561

16. Shahverdi AR et al (2007) Synthesis and effect of silver nanoparticles on the antibacterial activity of different antibiotics against Staphylococcus aureus and Escherichia coli. Nanomedicine 3:168-171

17. Fayaz AM et al (2010) Biogenic synthesis of silver nanoparticles and their synergistic effect with antibiotics: a study against Gram-positive and Gram-negative bacteria. Nanomedicine 6:103-109

18. Rai A, Prabhune A, Carole C (2010) Antibiotic mediated synthesis of gold nanoparticles with potent antimicrobial activity and their application in antimicrobial coatings. J Mater Chem 20:6789-6798

19. Rafii F, Shahverdi AR (2007) Comparison of essential oils from three plants for enhancement of antimicrobial activity of nitrofurantoin against Enterobacteria. Chemotherapy 53:21-25

20. Bowman MC et al (2008) Inhibition of HIV fusion with multivalent gold nanoparticles. J Am Chem Soc 130:6896-6897

21. Nomiya K, Noguchi R, Oda M (2000) Synthesis and crystal structure of coinage metal (I) complexes with tetrazole (Htetz) and triphenylphosphine ligands, and their antimicrobial activities. A helical polymer of silver (I) complex [Ag (tetz)(PPh3)2]n and a monomeric gold (I) complex [Au(tetz)(PPh3)]. Inorg Chim Acta 298:24-32

22. Nomiya K et al (2000) Synthesis, crystal structure and antimicrobial activities of two isomeric gold(I) complexes with nitrogencontaining heterocycle and triphenylphosphine ligands, $[\mathrm{Au}(\mathrm{L})$ $(\mathrm{PPh} 3)]$ (HLspyrazole and imidazole). J Inorg Biochem 78:363370

23. Noguchi R, Hara A, Sugie NK (2006) Synthesis of novel gold(I) complexes derived by $\mathrm{AgCl}$-elimination between [ $\mathrm{AuCl}(\mathrm{PPh} 3)]$ and silver(I) heterocyclic carboxylates, and their antimicrobial activities. Molecular structure of $[\mathrm{Au}(\mathrm{R}, \mathrm{S}-\mathrm{Hpyrrld})(\mathrm{PPh} 3)]$ (H2pyrrld2-pyrrolidone-5-carboxylic acid). Inorg Chem Commun 9:355-359

24. Banoee $M$ et al (2010) The green synthesis of gold nanoparticles using the ethanol extract of black tea and its tannin free fraction. Iran J Mater Sci Eng 7:48-53

25. Henglein A (1993) Physicochemical properties of small metal particles in solution: "microelectrode" reactions, chemisorption, composite metal particles, and the atom-to-metal transition. J Phys Chem B 97:5457-5471 
26. Sastry M et al (1997) pH dependent changes in the optical properties of carboxylic acid derivatized silver colloidal particles. Colloid Surf A 27:221-228

27. Sastry M et al (1998) Electrostatically controlled diffusion of carboxylic acid derivatized silver colloidal particles in thermally evaporated fatty amine films. J Phys Chem B 102:14041410

28. Nirmala Grace A, Pandian k (2007) Antibacterial efficacy of aminoglycosidic antibiotics protected gold nanoparticles: a brief study. Colloid Surface A 297: 63-70

29. Burygin GL et al (2009) On the enhanced antibacterial activity of antibiotics mixed with gold nanoparticles. Nanoscale Res Lett 4:794-801

30. Marques LL et al (2007) New gold(I) and silver(I) complexes of sulfamethoxazole: synthesis, X-ray structural characterization and microbiological activities of triphenylphosphine(sulfamethoxazolato-N2)gold(I) and (sulfamethoxazolato)silver(I). Inorg Chem Commun 10:1083-1087
31. Teitzel GM, Parsek MR (2003) Heavy metal resistance of biofilm and planktonic Pseudomonas aeruginosa. Appl Environ Microb 69:2313-2320

32. Fowles CC, Smoak EM, Banerjee IA (2010) Interactions of zeatin with gold ions and biomimetic formation of gold complexes and nanoparticles. Colloid Surf B 78:250-258

33. Chudasama B et al (2009) Enhanced antibacterial activity of bifunctional Fe3O4-Ag core-shell nanostructures. Nano Res 2:955-965

34. Aendekerk S, Ghysels B, Cornelis P, Baysse C (2002) Characterization of a new efflux pump, MexGHI-OpmD, from Pseudomonas aeruginosa that confers resistance to vanadium. Microbiology 148:2371-2381

35. Campbell SC et al (2001) Biogenic production of cyanide and its application to gold recovery. J Ind Microbiol Biotechnol 26 (3):134-139

36. Durán N (2007) Antibacterial effect of silver nanoparticles produced by fungal process on textile fabrics and their effluent treatment. J Biomed Nanotechnol 3:203-208 